\title{
Téoros
}

Revue de recherche en tourisme

\section{Costa Rica}

\section{La « Mecque » du tourisme gai en Amérique centrale}

\section{Dino Starcevic}

Volume 19, numéro 2, été 2000

Le tourisme des gais et des lesbiennes

URI : https://id.erudit.org/iderudit/1071964ar

DOI : https://doi.org/10.7202/1071964ar

Aller au sommaire du numéro

Éditeur(s)

Université du Québec à Montréal

ISSN

0712-8657 (imprimé)

1923-2705 (numérique)

Découvrir la revue

\section{Citer cet article}

Starcevic, D. (2000). Costa Rica : la « Mecque » du tourisme gai en Amérique centrale. Téoros, 19(2), 45-48. https://doi.org/10.7202/1071964ar

Ce document est protégé par la loi sur le droit d'auteur. L'utilisation des services d'Érudit (y compris la reproduction) est assujettie à sa politique d'utilisation que vous pouvez consulter en ligne.

https://apropos.erudit.org/fr/usagers/politique-dutilisation/
Cet article est diffusé et préservé par Érudit.

Érudit est un consortium interuniversitaire sans but lucratif composé de l'Université de Montréal, l'Université Laval et l'Université du Québec à Montréal. Il a pour mission la promotion et la valorisation de la recherche. https://www.erudit.org/fr/ 


\section{LA « Mecque » du tourisme gal en Amérique Centrale}

\section{Dino Starcevic}

Le Costa Rica décidait il y a quelques années de promouvoir le tourisme et d'en faire une de ses sources de revenus principales. Depuis, tant les organismes publics que privés du pays y ont consacré une part considérable de leurs énergies. Cette promotion du tourisme a coüncidé avec l'essor de la communauté gaie' et de son marché. Une telle expansion, cependant, a suscité certaines contradictions au sein de la société costaricaine, qui parie sur le tourisme, mais qui entretient toujours des craintes au sujet des gais et des lesbiennes. De telles contradictions, bien que non déterminantes, refont surface de temps en temps, rappelant que l'homophobie est bien présente, même au sein d'une société dont le seuil de tolérance est supérieur à celui d'autres sociétés latino-américaines.



Le Costa Rica : paradis de l'écotourisme...
Pour le Costa Rica, le tourisme semble constituer un pari sûr. Mais le jeu se corse quand vient le temps d'inclure les gais et les lesbiennes. Le pays possède des ressources de premier ordre : réputation de pays pacifiste et tolérant, population dont le niveau de scolarité est fort supérieur à la moyenne latino-américaine, infrastructures de communications sans cesse renforcées depuis trente ans et territoire riche en matières premières d'excellente qualité (depuis sa population jusqu'à ses richesses écologiques naturelles).

Depuis bientôt deux décennies, le tourisme au Costa Rica se trouve en plein processus d'expansion. Avant 1980, le Costa Rica comptait sur l'agriculture en tant que support économique principal, surtout sur l'industrie du café, à la base du développement de l'économie nationale depuis le $\mathrm{XIX}^{\mathrm{e}}$ siècle, ainsi que sur la culture de la banane, apparue au pays dans la première moitié du XX $\mathrm{X}^{\mathrm{e}}$ siècle à la faveur de l'instauration d'enclaves agricoles appartenant à des transnationales américaines. Même si le développement de l'industrie au sens strictement traditionnel du mot a été l'un des idéaux portés par les gouvernements des années 1960 et 1970, il est devenu évident que le Costa Rica était en mesure d'exploiter ce que l'on a convenu d'appeler $l^{\prime}$ ' industrie sans cheminées », c'est-à-dire le tourisme, grâce aux avantages comparatifs dont le pays jouit dans ce domaine.

Le Costa Rica se trouve parmi les pays latino-américains ayant des atouts supérieurs pour le tourisme : $65 \%$ de son périmètre territorial (1 290 kilomètres) correspond à des côtes aux plages et aux ressources maritimes impressionnantes ; il compte sur une flore et une faune parmi les plus riches de la zone tropicale des Amériques (en fait, c'est la zone de transition entre les régions Nord et Sud du continent), avec 12000 espèces de plantes, 237 espèces de mammifères, 848 espèces d'oiseaux, 361 espèces d'amphibiens et de reptiles. Sur un territoire d'à peine 51100 kilomètres carrés, ces espèces sont associées à diverses communautés vivant dans des climats particuliers, qui vont de la forêt tropicale humide jusqu'à la savane et au désert, dans une succession de microclimats d'une variété remarquable. Le réseau des parcs nationaux, l'un des plus avantgardistes de la région, comprend 28 aires protégées (524 917 hectares, soit 10,27\% du territoire national). Tout cela a fait en sorte que le tourisme, grâce à une volonté politique officielle constamment soutenue par des campagnes et des investissements, est devenu la troisième source - peut-être même la deuxième - de revenus nationaux.

\section{TOURISME ET CONJONCTURE}

La décision officielle de tirer profit de ces avantages en faisant la promotion du tourisme (et, récemment, de l' «écotourisme » en particulier, parallèlement au tourisme traditionnel de haute qualité offrant hôtels cinq étoiles avec plage et tous les services complémentaires) a été bien accueillie par la communauté et par l'entreprise privée, qui ont consacré beaucoup d'énergie afin d'en bénéficier. En effet, $49 \%$ de la population économiquement active $œ u v r e$ dans le secteur des services, dont une partie significative en tourisme. 
La communauté lgbt n'est pas restée à l'écart de ce phénomène, ce qui est dû surtout au fait que la promotion du tourisme a coïncidé avec la montée de la prise de conscience et de la lutte pour les droits des lesbiennes, des gais, des bisexuels et des transsexuels. Même si l'existence d'un milieu gai et lesbien était une réalité avant les années 1980, l'apparition et la propagation du sida ont enclenché des transformations remarquables au Costa Rica. C'est alors que les premiers groupes et organismes communautaires se sont activés, s'adonnant à l' analyse de la situation des gais, des lesbiennes, des bisexuels et des transsexuels au pays, au développement de campagnes massives de prévention et à l'ouverture d'espaces politiques et juridiques qui ont facilité l'émergence de lieux de réunion et d'établissements commerciaux de plus en plus élaborés.

En 1998-1999, une étude de la population gaie du Costa Rica, réalisée par l'Institut latino-américain pour la prévention et l'éducation en santé (ILPES) en collaboration avec d'autres institutions régionales, a permis de dresser un portrait général de la clientèle de ces services et de ces commerces ; il s'agit d'individus pour la plupart jeunes (de 20 à 34 ans), dont le niveau de scolarité se situe entre l'école secondaire et l'université, célibataires (86\%), sans enfants $(83 \%)$, à faible religiosité (60\% déclarent être peu ou pas religieux) quoique, à l'image du pays dans son ensemble, lorsqu'ils sont pratiquants ils se déclarent catholiques (63\%).

Grâce à son système d'éducation universel et aux valeurs de respect démocratique et juridique dont le développement remonte aux débuts de l'indépendance (milieu du $\mathrm{XIX}^{\mathrm{e}}$ siècle), l'environnement tolérant qui caractérise le pays fait du Costa Rica un cas à part en Amérique centrale, ce dont la communauté lgbt a bénéficié. Sur une telle base et par le biais de diverses organisations et initiatives privées, elle a ainsi progressé comme nulle autre dans la région centraméricaine.

La lutte politique amorcée par les organisations a contribué à l'amélioration des conditions de vie des gais, des lesbiennes, des bisexuels et des transsexuels, grâce à des démarches judiciaires qui ont établi le respect des droits individuels face à l'action gouvernementale. À la différence de son voisin, le Nicaragua (où l'homosexualité est actuellement illégale), le Costa Rica a éliminé en 1970 le statut criminel jadis réservé aux actes homosexuels. Les descentes policières dans les sites de réunion de gais ont été interdites au début de la dernière décennie. Les arrestations non justifiées de personnes (y compris les travestis s'adonnant à la prostitution) ont été déclarées illégales. En outre, résultat des campagnes de sensibilisation au sida, la notion même de l'existence d'une communauté lgbt au pays a été comprise par la société dans son ensemble.

Le boom économique de la communauté lgbt a commencé à se faire sentir il y a environ dix ans. Il y a toujours eu des lieux de réunion tels les bars et les discothèques qui, au Costa Rica, étaient traditionnellement concentrés dans la capitale, San José, mais depuis les années 1990, ces activités se sont sensiblement diversifiées. L'élimination de la répression policière à l'égard des endroits de réunion a permis aux bars et aux discothèques de s'afficher, laissant derrière leur ancienne clandestinité. De nos jours, les discothèques gaies et lesbiennes sont listées dans les pages des guides touristiques et sont connues du public et de toutes les entreprises (les chauffeurs de taxi par exemple). Des espaces consacrés à la culture, à l' art et aux variétés existent (actuellement, les spectacles et les concours de travestis sont monnaie courante) et on a mis sur pied des établissements tels des cafétérias et des restaurants gais ou gay friendly, de sorte que l'offre s'est diversifiée et ne se limite plus aux seuls bars et discothèques.

Il existe dans la capitale, mais aussi dans divers points du territoire national, une industrie hôtelière destinée à la communauté lgbt et des entreprises de services touristiques affiliées. Au sud-ouest du pays, une véritable « Mecque » gaie s'est développée, sur les plages de Manuel Antonio qui sont régulièrement visitées par des milliers d'hommes gais, autant costaricains qu'étrangers. Tout autour, la zone qui comprend un parc national et une ville, Quepos, a vu fleurir toute une industrie touristique destinée à la communauté lgbt. Le plus nouveau dans cette expansion des services, c'est l'apparition de saunas gais dans la capitale. Bien que ce type de commerce ait existé depuis des décennies, les saunas les plus récents ont laissé derrière la tradition de clandestinité pour s'établir dans les zones centrales de la ville, offrant des services de qualité et attirant une clien- tèle toujours croissante issue des strates sociales les plus diverses. Parallèlement à ces développements, des médias ont vu le jour ; ils maintiennent la communauté bien informée en tout temps. Une revue (Gente 10) et un périodique mensuel (Gayness) circulent au Costa Rica et un véritable réseau de contacts profite de l'essor d'Internet, réseau agglutiné autour du site de Gay Costa Rica (http://www. gaycostarica.com).

\section{DES DÉCISIONS DE POIDS}

En dépit de ses remarquables atouts, la situation au Costa Rica est loin d'être parfaite. L'homophobie est un fait courant. La répression officielle a été contrôlée grâce aux tribunaux, mais il y a toujours des signes qui indiquent qu'elle peut à nouveau se déployer. Il y a quelques mois, le maire de San José entreprenait une démarche répressive à l'endroit d'un des nouveaux saunas gais, affirmant se porter à la défense des principes moraux et prétextant l'absence d'un permis d'exploitation que, par ailleurs, les autorités ellesmêmes refusaient au propriétaire du sauna. Ces tentatives ont toutefois échoué quand les tribunaux ont donné raison au propriétaire.

La lutte a déjà une histoire. Au début des années 1990, les autorités ont essayé d'entraver la réalisation au Costa Rica d'un congrès lesbien qui allait réunir des femmes en provenance de divers points de l'Amérique latine pour discuter des droits des lesbiennes. Bien que ce congrès ait été organisé comme activité privée, le gouvernement a fait tout ce qui était en son pouvoir pour en empêcher la réalisation : depuis le refus des visas d'entrée au pays à des femmes «suspectes », les autorités sont allées jusqu'à répudier publiquement l'événement dans les médias. Par ailleurs, il y a moins de deux ans, le pays entier se mobilisait (tant pour que contre) quand on a annoncé la tenue d'une réunion de gais de diverses origines. Même s'il s'agissait d'un congrès d'entrepreneurs et de clients potentiels du secteur touristique gai, le discours tenu à cet égard dans divers milieux (surtout au sein de l'Église catholique) présentait l'événement comme une rencontre caractérisée par la dégénérescence homosexuelle qui aurait des effets négatifs sur les communautés vivant sur les lieux de la réunion. 
La polémique a éclaté avec une telle force qu'elle a atteint les plus hautes sphères, y compris le Président de la république et l'Archevêque de San José, chef de l'Église, qui se sont publiquement et ouvertement prononcés contre le congrès, leur discours comportant une haute teneur homophobe. À la suite de ces déclarations, des personnes et des organisations liées à la tenue de l'événement ont entamé des poursuites judiciaires contre les deux hommes. Au point culminant de la saga, une organisation pro droits humains amenait l'Archevêque, M. Román Arrieta, devant les tribunaux, l'accusant de discrimination. Ce dernier a toutefois été absous de ces accusations.

Ces démarches démontrent deux choses : que l'homophobie existe et que la lutte en faveur des droits lgbt - non seulement leurs droits humains fondamentaux, mais également leurs droits économiques et commerciaux - est loin d'être gagnée. Malgré les atouts du Costa Rica en matière de promotion du tourisme et du commerce lgbt, il est clair que la lutte et l'éducation sont toujours nécessaires pour que les deux activités fonctionnent sans heurts.

De plus, la profonde contradiction qui persiste par rapport au tourisme gai devient de la sorte évidente, du moins parmi les autorités officielles. Un État qui, de façon consciente et intentionnelle, a décidé de promouvoir le tourisme en tant qu'industrie de haute priorité pour le développement du pays, réprime en même temps certaines manifestations d'une telle industrie parce qu'il les considère « contraires » à la morale. En d'autres mots : le tourisme et bon, mais le tourisme gai ne l'est pas au même titre. Bien entendu, l'excuse brandie pour établir la distinction est le sexe. Dans divers paliers officiels, le tourisme gai est considéré comme l'équivalent du tourisme sexuel, dont il faudrait mettre le Costa Rica à l'abri.

C'est dans un tel contexte que la naissante industrie touristique lgbt costaricaine doit se soucier d'affirmer clairement ses propres objectifs. Le danger du tourisme sexuel, compris surtout comme exploitation des gens du pays, est une réalité dans différentes régions de la planète et le Costa Rica n'est pas une exception. Il est facile - et les exemples abondent - de céder à la tentation d'attirer les touristes (en particulier les hommes gais) en leur présentant des images d'hommes légèrement

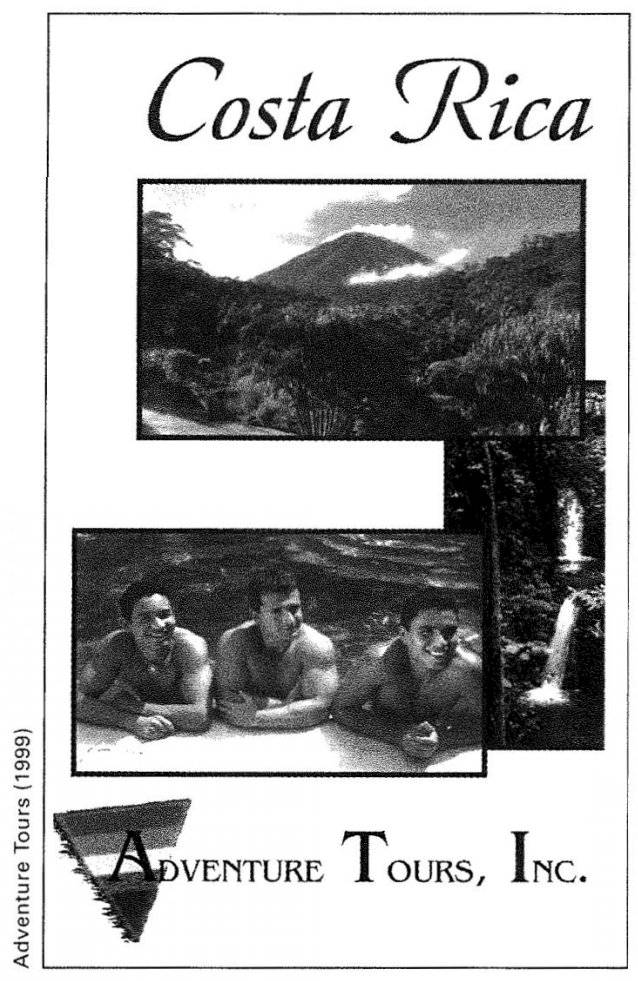

Brochure promotionnelle gaie.

vêtus sur fond de paysage paradisiaque, des images suggérant que les gais costaricains sont « disponibles » pour satisfaire les fantasmes des visiteurs. Un tel appât commercial est actuellement utilisé autant par des entreprises étrangères que nationales. La réalité, bien entendu, est tout autre.

\section{ÊTRE GAI AU COSTA RICA}

Faire la rencontre, en tant que touriste, de la communauté lgbt au Costa Rica requiert la compréhension de quelques-uns de ses traits en tant que groupe enraciné dans une nation. La personnalité collective des gais au Costa Rica est profondément marquée par le développement historique et social du pays. Les Costaricains ont été traditionnellement dépeints comme des gens éduqués, tolérants et paisibles, ouverts aux influences étrangères ; quant à leur côté moins aimable, il est lié à leur passé de nation agricole peu peuplée et éloignée des grands centres de pouvoir, renfermée dans un environnement montagnard, ce qui fait d'eux des gens réservés et peu enclins aux manifestations visibles de joie ou de fête, à la différence des nations qui les avoisinent, comme le Nicaragua et le Panama, dont les habitants sont reconnus pour leur tempérament extraverti. De tels facteurs historiques ont fait en sorte que soit cultivée au Costa Rica, de manière non officielle, une idée de « supériorité » par rapport aux voisins centraméricains. Une telle idée s'appuie non seulement sur des questions sociales telles la démocratie et le haut niveau de scolarité, mais aussi sur des considérations raciales : pendant des décennies, la « mythologie » nationale dépeignait les Costaricains comme descendants des Espagnols ou des Européens au milieu d'une région éminemment indigène. Cette idée de supériorité a provoqué de nombreux conflits avec le reste des Centraméricains, qui perçoivent les Costaricains comme étant isolationnistes et suffisants. Tous ces traits nationaux sont présents au sein de la communauté lgbt. En termes généraux, on peut remarquer en elle une plus forte disposition à la lutte collective en faveur de ses propres droits, grâce au labeur et à l'éducation réalisés par des organisations qui travaillent à éveiller une conscience lgbt au pays. Il est plus facile de trouver un citoyen prêt à se reconnaître homosexuel et à défendre ses droits en tant que gai ou lesbienne au Costa Rica qu'ailleurs en Amérique centrale.

Un contact soutenu avec des communautés et des processus lgbt dans des pays développés (en particulier aux États-Unis) a fait en sorte que plusieurs de leurs caractéristiques se reproduisent au Costa Rica, dans le meilleur et le pire. Les gais costaricains, élevés dans cette idée de "supériorité » déjà mentionnée, font preuve d'une certaine tendance à cultiver leur image, sans cesse stimulés par les opinions des touristes, qui mettent l'accent sur la « beauté » du Costaricain. On note un culte hédoniste de la beauté et de la jeunesse qui devient fort évident dans les endroits où ils se réunissent, des discothèques jusqu'aux saunas. Par ailleurs, malgré la propagande officielle en matière d'égalité sociale, les différences entre les groupes économiques sont visibles. Certains bars ou discothèques à San José vont chercher des clients appartenant à des strates moyennes et riches, alors que d'autres groupes développent leurs propres établissements. Les lesbiennes tendent à s'isoler et à chercher leurs propres sites de réunion.

En tant que touristes dans leur propre pays, les Costaricains adorent les plages, tout particulièrement les plages de Manuel Antonio, zone qui accueille deux grandes 
«vagues » annuelles, à Pâques et en décembre. Guanacaste, au nord-est, et Limón, sur la côte caraibéenne, sont les deux autres destinations touristiques par excellence. Et la réputation des Costaricains en tant que voyageurs internationaux est constamment signalée dans les médias, les ÉtatsUnis étant leur destination préférée et la « Mecque » gaie de Miami se trouvant couramment à l'agenda de plusieurs.

Pour les touristes gais qui arrivent de l'extérieur (surtout les Américains et les Européens, quoique beaucoup de Centraméricains visitent le pays pour profiter de la liberté et de l'activité bouillonnante qu'ils ne trouvent pas chez eux), le Costa Rica offre un grand éventail d'activités, tant dans les villes que la nature. L'écotourisme, dont le développement a été fulgurant ces dernières années, n'est pas pour l'instant prioritaire chez les gais et les lesbiennes. Bien entendu, les offres sexuelles ne sont pas absentes. Nombreux sont les touristes (particulièrement des personnes d'âge avancé) qui viennent au Costa Rica attirés par l'idée d'y faire des rencontres romantiques faciles. Il y a au pays des endroits où l'échange sexuel n'est pas compliqué. L'ambiance à Manuel Antonio, où 1'on trouve une plage naturiste, est chargée d'érotisme. A San José il y a des endroits pratiquement destinés à cela, tels le Parc métropolitain de La Sabana ou le Parc national, en plein cœur de la ville, où la prostitution masculine est exercée au vu et au su de tous. Les saunas, d'autre part, connaissent un essor remarquable ; ce sont là des sites explicitement destinés aux rencontres sexuelles des utilisateurs. Mais ce niveau de sexualisation n'est ni supérieur ni plus visible qu'ailleurs dans le monde. La différence, peut-être, réside en ce que la tolérance qui caractérise les Costaricains leur permet de cohabiter avec ces phénomènes sans entreprendre de chasses aux sorcières à teneur moralisatrice.

Voici les cartes du jeu touristique gai costaricain. Si on se fie à l'évolution récente du phénomène, caractérisé par la consolidation du poids politique de la communauté gaie et lesbienne et par l'ouverture de la société à la diversité, il est à parier que tous les joueurs sortiront gagnants, tant au point de vue économique que social.

Traduit de l'espagnol par Roberto Jovel.
Journaliste et historien costaricain vivant à San José, Dino Starcevic est l'éditeur du mensuel Gayness. Il travaille à l'Institut latino-américain de prévention et d'éducation en santé (ILPES), organisation non gouvernementale cuvrant à la prévention du VIH/sida et à la promotion de l'accroissement du pouvoir de groupes minoritaires, dont la communauté lgbt. Il a été rédacteur, éditeur et chef de l'information du journal La República (Costa Rica) et éditeur régional de l'agence de presse Inter Press Service. Il a collaboré à la réalisation de diverses recherches publiées par la maison d'édition ILPES et par The Haworth Press. Il est l'auteur de Entendiendo la homosexualidad (Éd. ILPES). Il travaille présentement à la production d'un guide gai sur l'Amérique centrale.

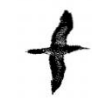

\section{NOTE}

1 L'auteur emploie le terme gai pour inclure à la fois les gais et les lesbiennes. Le terme lgbt est aussi employé pour refléter la diversité de la communauté lesbienne, gaie, bisexuelle, travestie et transsexuelle.

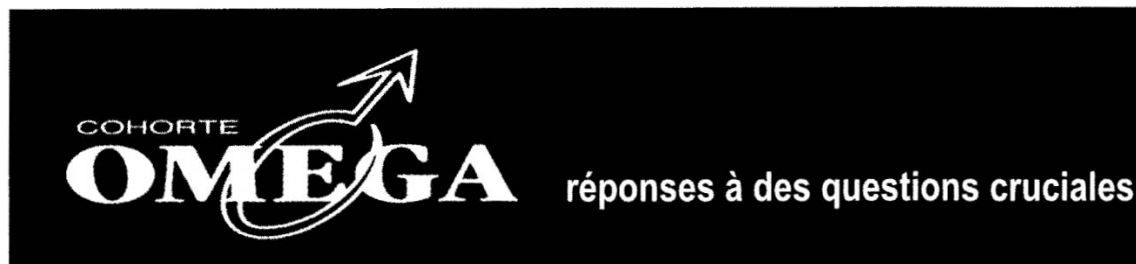

Malgré l'utilité des campagnes de prévention, qui ont déjà sauvé des milliers de vies, nous sommes forcés de nous demander pourquoi et comment tant d'hommes gais ou bisexuels contractent le VIH encore aujourd'hui.

A Montréal seulement, en 1999, près de 400 hommes gais ou bisexuels ont contracté le VIH. Également à Montréal depuis le début de l'épidémie dans les années 80 , plus de 1756 personnes sont décédées des suites du $\mathrm{VIH} /$ sida. Quels sont les contextes sociaux, les processus relationnels et psychologiques qui entourent linfection? Comment intervenir plus efficacement pour prévenir la transmission du VIH?
C'est pour répondre à ce type de questions qu'une équipe de chercheurs a conçu

OMÉGA, la plus vaste enquête psychosociale et épidémiologique jamais effectuée au Canada auprès d'hommes ayant des relations affectives et sexuelles avec d'autres hommes. La recherche OMÉGA suivra, sur plusieurs années, 2000 hommes séronégatifs âgés de 16 ans et plus, et qui vivent dans la région de Montréal.

OMÉca demeure à ce jour l'une des recherches les plus importantes jamais produites au monde. Elle vient s'ajouter à d'autres études similaires qui se déroulent notamment en Australie, en Angleterre et aux États Unis.

Pour information ou pour participer voici notre numéro : 514.528 .8565 